\title{
Nurturing the Future
}

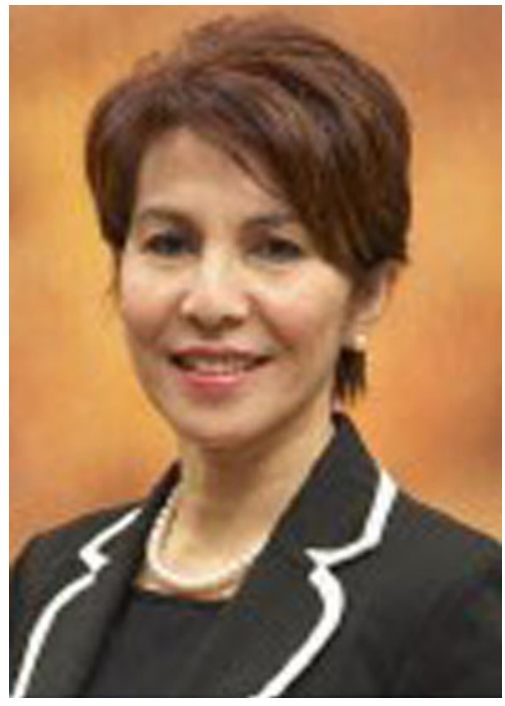

The clinician is faced with many issues in ensuring best practices for the management of the patient. Guidance on the appropriate approach of treatment must come from evidence based research combined with good clinical decision making. Relevant research highlighting cutting edge science on treatment methodologies, genetics, gene therapy, the impact of lifestyle changes and challenges in the environment should be published to enable knowledge dissemination for optimal patient management of even the most common oral maxillofacial condition.

Multi centre collaborative studies should be encouraged, multi-site cooperation essential, particularly in the developing world where resources are limited and access to care hampered by financial and environmental constraints.
The creation of an Asian database of craniofacial anomalies should be a significant agenda for the future of the Asian Association of Oral and Maxillofacial Surgeons. Through networking with other regional associations that have such registries in place, a consensus approach could be reached to standardise methodologies and protocols which will translate to more effective clinical practice. This ties in neatly with the need for standardisation of competency of practice of the Asian Association of Oral and Maxillofacial Surgeons through Board certification.

Such certification ensures credibility, benchmarking and quality control benefitting the profession.

The future of the specialty requires professionals with a clear vision to inspire cooperation. Progressive growth will only occur if dedicated individuals blaze the trail for others to change and follow nurturing the next generation of leaders.

Mahatma Gandhi once said "You must be the change you wish to see in the world"-words of wisdom that should inspire every individual to aspire to be the agent of change for the future through upholding the principles of compassion, integrity, dedication and commitment for excellence.

Congratulations to the Indian Association of Oral and Maxillofacial Surgeons on the publication of their journal. I am certain it will have a positive impact towards furthering an avenue for solid intellectual discourse for the oral and maxillofacial community.

Dr. Sharifah Fauziah Alhabshi President

Asian Association of Oral and Maxillofacial Surgeons. 